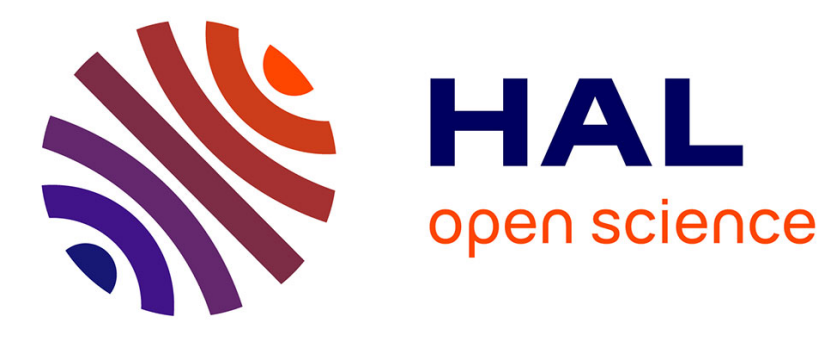

\title{
Les migrations internationales face aux nouvelles frontières de l'Europe
}

Raimondo Cagiano de Azevedo, Gérard-François Dumont

\section{To cite this version:}

Raimondo Cagiano de Azevedo, Gérard-François Dumont. Les migrations internationales face aux nouvelles frontières de l'Europe. Population et avenir, 2012, 709, pp.17-19. 10.3917/popav.709.0017 . halshs-00770092

\section{HAL Id: halshs-00770092 \\ https://shs.hal.science/halshs-00770092}

Submitted on 2 Dec 2019

HAL is a multi-disciplinary open access archive for the deposit and dissemination of scientific research documents, whether they are published or not. The documents may come from teaching and research institutions in France or abroad, or from public or private research centers.
L'archive ouverte pluridisciplinaire HAL, est destinée au dépôt et à la diffusion de documents scientifiques de niveau recherche, publiés ou non, émanant des établissements d'enseignement et de recherche français ou étrangers, des laboratoires publics ou privés. 


\section{Les migrations internationales face aux nouvelles frontières}

\section{de l'Europe}

Le champ d'étude des migrations internationales apparaît immuable. Il consiste à examiner les migrations résidentielles de personnes ayant passé une frontière internationale. Il permet aussi de mettre en évidence l'existence de frontières démographiques. Mais, tout particulièrement en Europe, il impose une pensée renouvelée dans la mesure où les frontières s'avèrent mouvantes et variées en raison de changements dans la géographie politique. Il faut donc dresser une typologie des frontières qui engendre des natures fort différentes de migrations internationales.

\section{Frontières politiques et frontières démographiques}



a science de la population enseigne que les frontières ne sont pas seulement géopolitiques. Elles peuvent être aussi démographiques. L'analyse des populations met en évidence la réalité des frontières internationales, mais aussi de frontières infranationales. Par exemple, l'examen de deux pays frontaliers montre souvent des caractéristiques différentes de natalité ou de mortalité entre les deux pays considérés. Ainsi, la frontière entre la Pologne et la Lituanie, qui sépare pourtant deux pays membres de l'Union européenne depuis la même date, le $1^{\text {er }}$ mai 2004, est une frontière démographique, avec par exemple, une espérance de vie à la naissance des hommes nettement plus faible en Lituanie qu'en Pologne ${ }^{1}$. À l'inverse, d'autres différences démographiques nient les frontières politiques, par exemple lorsque des frontières historiques résistent aux changements géopolitiques. Ainsi la province italienne de Bolzano, au nord de l'Italie, a des caractéristiques démographiques assez différentes de celles de l'Italie et plus proches de celles de cette Autriche avec laquelle elle a longtemps été politiquement intégrée dans l'empire austro-hongrois.

\section{Besoin de services aux personnes} âgées et immigration féminine

D’autres frontières démographiques sont liées à la spécificité de la composition par sexe ou par âge des populations. Lorsque de telles spécificités engendrent des migrations internationales, la démographie contribue évidemment à témoigner de la réalité des frontières. Considérons la question du vieillissement de la population, en nous fondant sur le cas de l'Italie ${ }^{2}$. Le vieillissement de la population de ce pays ${ }^{3}$ est aisé à comprendre. Il tient à deux facteurs. D'une part, il résulte de l'abaissement de la fécondité, de «l'hiver démographique " qui réduit la taille moyenne des familles, ce qu'on appelle le vieillissement « par le bas ». D’autre part, il provient de l'augmentation de l'espérance de vie des personnes âgées, ce qu'on appelle le vieillissement « par le haut».

Or le vieillissement de la population de l'Italie s'accompagne d'une augmentation du nombre de personnes âgées, c'est-àdire d'une gérontocroissance ${ }^{4}$ et, en même temps, d'une baisse de la population active. En théorie, le système de protection sociale doit soutenir les personnes âgées par le biais de retraites, de soins à domicile ou de divers services appropriés. Comme il faut du personnel pour satisfaire les personnes âgées, le nombre de travailleurs dans ce domaine doit augmenter avec la gérontocroissance. Or la baisse des Italiens dans la population active ne pouvant fournir ce personnel, nombre de personnes qui travaillent dans ce secteur des services aux personnes âgées sont des femmes nées à l'étranger, souvent d'origine ukrainienne ou moldave.

\section{LA PYRAMIDE DES ÂGES}

DE LA POPULATION UKRAINIENNE

\section{RÉSIDENTE EN ITALIE}

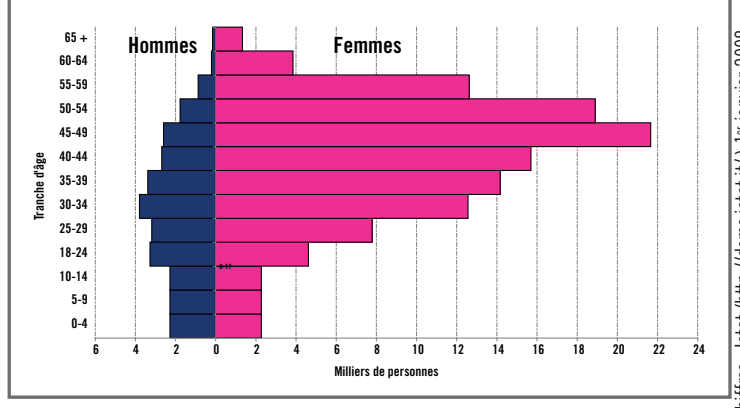

Des flux migratoires internationaux sont donc la conséquence du phénomène démographique décrit ci-dessus, la gérontocroissance. Ils sont une réponse démographique à une question d'ordre démographique. En examinant la pyramide des âges de la population ukrainienne résidente en Italie, une proportion élevée de femmes âgées de 40 à 55 ans apparaît. Cette proportion remarquable correspond à la demande de femmes ukrainiennes en Italie pour les services aux personnes âgées. Cette migration a été encouragée par les deux pays, l'Italie demandant de la main-d'œuvre et l'Ukraine appréciant de percevoir des transferts d'argent venant de leurs ressortissants employés en Italie. par Raimondo CAGIANO de AZEVEDO* et Gérard-François DUMONT**

* Université de Rome "La Sapienza".

** Université de ParisSorbonne.

1. Elzbieta GrzelakKostulska, Beata Holowiecka, Hanna Holowiecka, Hanna
Michniewicz-Ankiersztajn, «Europe : de grands écarts dans l'espérance de vie ", Population \& Avenir, $n^{\circ} 706$, janvierfévrier 2012.

2. Cf. également : Terranova, Giuseppe, "L'attractivité de I'Italie : diversité territoriale et politique nationale ", Population \& Avenir, $n^{\circ}$ 698, maijuin 2010.

3. Cette tendance au vieillissement de la population en Italie, qui se manifeste dans l'ensemble de l'Europe depuis le milieu des années 1970, se constate ágalement dans de egalement dans de nombreuses autres régions du monde. II est par exemple fort intense en Chine ; Cf. Dumont, Gérard-François, "Les six enjeux de la démographie chinoise ", Géostratégiques, $n^{\circ} 33$, 4 e trimestre 2011.

4. Dumont, GérardFrançois et alii, Les territoires face au vieillissement, Paris, Ellipses, 2006. 


\section{Besoins économiques et migrations mascullines}

5. « Est citoyen de I'Union toute personne ayant la nationalité d'un État membre ».

6. Pensons à celui entr Menton (Alpes-Maritimes) Menton (Alpes-Maritimes) et Vintimille (Ligurie) qui, aux heures d'affluence, créait un embouteillage. 7. Même si les statistiques de l'Onu continuent à les continuent à les
dénommer « immigrants internationaux ». internationaux ». 8. Dumont, GerardFrançois, Verluise, Pierre, Géopolitique de l'Europe, Paris, Sedes, 2009

Le cas de l'Italie offre l'exemple de migrations correspondant à d'autres compositions par sexe. L'économie italienne, confrontée à une baisse de population active ou à une faible appétence de ses nationaux pour certains métiers, a besoin de main-d'œuvre. Ainsi la population tunisienne résidente en Italie, qui travaille notamment dans l'agriculture ou la pêche, a une pyramide inverse de

\section{LA PYRAMIDE DES ÂGES}

\section{DE LA POPULATION TUNISIENNE} RÉSIDENTE EN ITALIE

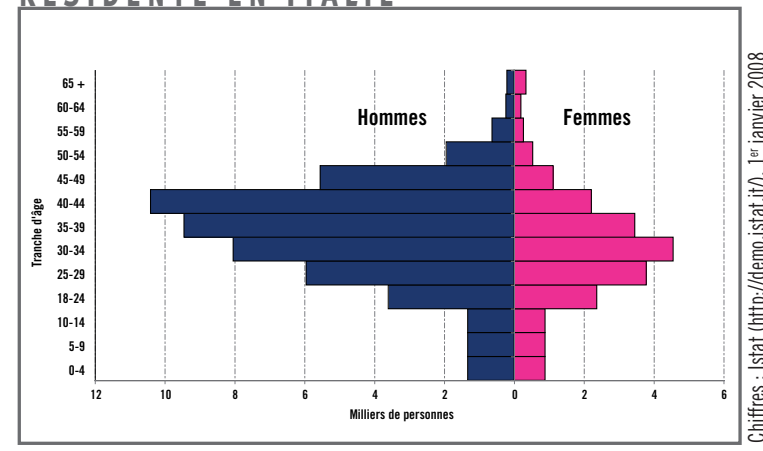

celle des Ukrainiens avec une très forte proportion des hommes. À l'instar des Tunisiens, il y a aussi une forte proportion d'hommes, assez jeunes, dans l'immigration marocaine et dans l'immigration sénégalaise, cette dernière travaillant notamment dans le commerce ambulant. C'est donc bien la situation démographique, telle qu'elle se présente en Italie, qui, marquant une frontière dans des besoins sur le marché italien de l'emploi, explique certaines migrations internationales.

\section{L'évolution géographique des frontières, marqueur des migrations internationales}

La question de la frontière est ainsi au cœur de l'analyse migratoire. Toutefois, il est banal de dire qu'il n'y aurait pas de migrants internationaux s'il n'y avait pas de frontières. Autrement dit, la démographie s'intéresse à la question des frontières parce qu'il y a des migrants internationaux et pas seulement des migrants internes aux pays. Toutefois, les conséquences de ce qui peut apparaître comme une banalité ne sont pas toujours évidentes. D'une part, toute création d'une nouvelle frontière, donc toute modification de la géographie des frontières, transforme des migrations internes en migrations internationales. Par exemple, l'éclatement de la Yougoslavie a fait que les migrations entre Zagreb, capitale de la Croatie, et Belgrade, capitale de la Serbie, auparavant de nature interne, sont devenues des migrations internationales.

\section{Migrants internationaux ou citoyens en mobilité}

D'autre part, tout affaiblissement juridique significatif d'une frontière pour certaines personnes, c'est-à-dire toute modification de géographie politique relativisant la séparation politique qu'illustre la frontière, peut changer la nature de leur migration. Ainsi, avant l'instauration par les traités européens de la libre circulation des personnes entre l'Italie et la France et l'entrée de l'Italie dans l'espace Schengen en 1997, les personnes de nationalité italienne résidant en France, comme les personnes de nationalité française résidant en Italie, pouvaient être considérées comme des immigrants internationaux qui avaient fait l'objet d'un double contrôle douanier - français et italien - au moment du passage de la frontière. Depuis l'instauration de la citoyenneté européenne introduite par le traité de Maastricht de 1992 pour les nationaux de ces deux pays ${ }^{5}$ et surtout la suppression des contrôles à la frontière au sein de l'espace Schengen, qui s'est accompagné du démantèlement des postes de douanes ${ }^{6}$, ces personnes sont désormais des citoyens en mobilité, totalement libres de se déplacer et de travailler au sein d'un espace commun'. L'instauration d'accords internationaux ou de traités peut donc changer l'analyse de la mobilité des populations.

\section{La nature des migrations liée aux changements réglementaires}

La question des migrations n'est pas donc exclusivement liée aux choix des migrants eux-mêmes, aux facteurs expliquant leur migration, mais aussi aux évolutions du cadre juridique et économique national et international qui peut affecter ou modifier la nature juridique de leur migration. Ainsi, le Polonais qui émigrait sans autorisation de son État en direction de la France en 1985 commettait un délit ; le Polonais qui émigrait en direction de la France en 2006 sans y prendre un emploi circulait librement au sein d'un espace commun de libre circulation des hommes. Mais, toujours en 2006 , le travailleur polonais qui venait occuper un emploi en France sans autorisation administrative commettait aussi un délit car la liberté de circulation des travailleurs entre la Pologne et la France n'était pas encore instaurée ${ }^{8}$. En 2012, le travailleur polonais qui vient occuper un emploi en France ne fait que se déplacer au sein d'un espace communautaire de libre circulation des hommes et des travailleurs. Un nouveau traité international (soit le traité d'adhésion de la Pologne à l'UE) ou son application par les Etats (en l'espèce la fin de la période probatoire que la France avait imposée aux Polonais et qui empêchait la liberté de circulation des travailleurs entre la France et la Pologne) peut faire passer de la situation d'immigrant en situation irrégulière à celle de simple citoyen en mobilité.

\section{La multiplication des types de frontières}

Mais, pour la démographie, analyser les effets des frontières ne doit pas se limiter à considérer les frontières politiques que présentent les cartes. D'abord, l'étude des migrations internationales suppose de se demander si ces frontières sont militairement fermées, comme entre la Corée du Nord et la Corée du Sud, ou, à l'opposé, considérablement ouvertes, comment entre la France et l'Italie.

Ainsi, au sein de l'UE, il faut distinguer plusieurs types de frontières extérieures. Le premier type concerne les frontières entre des pays pleinement membres de l'UE. Ces 
frontières marquent certes des séparations au regard du système institutionnel ou des modes d'élection. Mais elles n'existent pas au regard de très nombreuses réglementations qui s'appliquent de la même façon de part et d'autre des frontières de deux pays membres de l'UE. Cette organisation régionale ${ }^{9}$ est, on le sait, l'expérience d'intégration régionale la plus avancée dans le monde, intégration dont le support juridique est formé de l'ensemble des traités et des réglementations communes que ses membres ont adoptés depuis le traité de Rome de 1957, ensemble que l'on appelle l'« acquis communautaire ». Cet acquis engendre une frontière spécifique entre les pays membres de l'UE et ceux qui n'en sont pas membres. D'ailleurs, tout pays qui a souhaité adhérer à l'UE (ou qui souhaite y adhérer) a dû (ou doit) d'abord franchir cette frontière, c'est-àdire modifier l'ensemble de ses législations pour se conformer à l'acquis communautaire.

L'Europe connaît deux autres types de frontières, d'une autre nature, selon que les membres de l'UE sont ou non membres de l'espace Schengen. Ainsi, le Français qui va en Italie n'a pas de contrôle douanier à la frontière alors le Français qui se rend au Royaume-Uni passe un double contrôle douanier, français et britannique, puisque le Royaume-Uni n'est pas membre de l'espace Schengen ${ }^{10}$. Un quatrième type de frontière apparaît entre des pays membres de l'UE et des pays non membres, mais toutefois adhérents à l'espace Schengen, comme entre l'Italie et la Suisse, ce dernier pays étant entré dans cet espace Schengen en 2008.

L'espace euro forme encore un nouveau type de frontière puisqu'il s'agit d'une frontière qui exclut toute nécessité de recourir à des opérations de change. II faut ajouter qu'il existe encore d'autres types juridique de frontières entre les pays de l'Espace économique européen (EEE), né en 1994, précisément entre ceux qui sont membres de l'UE et d'autres membres de l'Association européenne de libreéchange AELE (Islande, Liechtenstein et Norvège), entre les pays de l'UE et ceux qui ont les statut de pays candidats (Turquie, Macédoine...), entre les pays de l'UE et ceux qui ont un statut dit avancé comme le Maroc, entre les pays de l'UE et ceux qui relèvent de la politique européenne de voisinage, développée depuis 2004 pour les pays voisins de l'UE (Tunisie, Liban, Ukraine...), pays avec lesquels l'UE est prête à partager « tout sauf les institutions $» .$.

\section{Une notion en profonde évolution}

Ainsi, les frontières des pays européens ont changé et la notion de "migrations internationales » doit évoluer. Les frontières de l'Europe reposent souvent sur une définition géographique et politique, parfois encore considérée par convention, prenant en compte d'une part le détroit de Gibraltar ou la chaîne du Caucase et, d'autre part, les frontières nationales issues de l'histoire et incluant parfois des réalités géographiques, comme ces lignes de partage des eaux qui séparent la France et l'Espagne ou la Suède et la Norvège. Aujourd'hui, les frontières de l'Europe prennent en compte une forte dimension juridique. D'un côté, des pays du sud Caucase, généralement classés dans l'Asie occidentale, sont membres du Conseil de l'Europe, alors que la Biélorussie, trop peu respectueuse de l'État de droit, n'en fait pas partie. De l'autre, la citoyenneté délivrée aux nationaux des pays de l'UE, citoyenneté qui

\section{LES FRONTIÈRES DIFFÉRENCIÉES}

\section{DE L'UNION EUROPÉENNE} ET DE L'ESPACE SCHENGEN

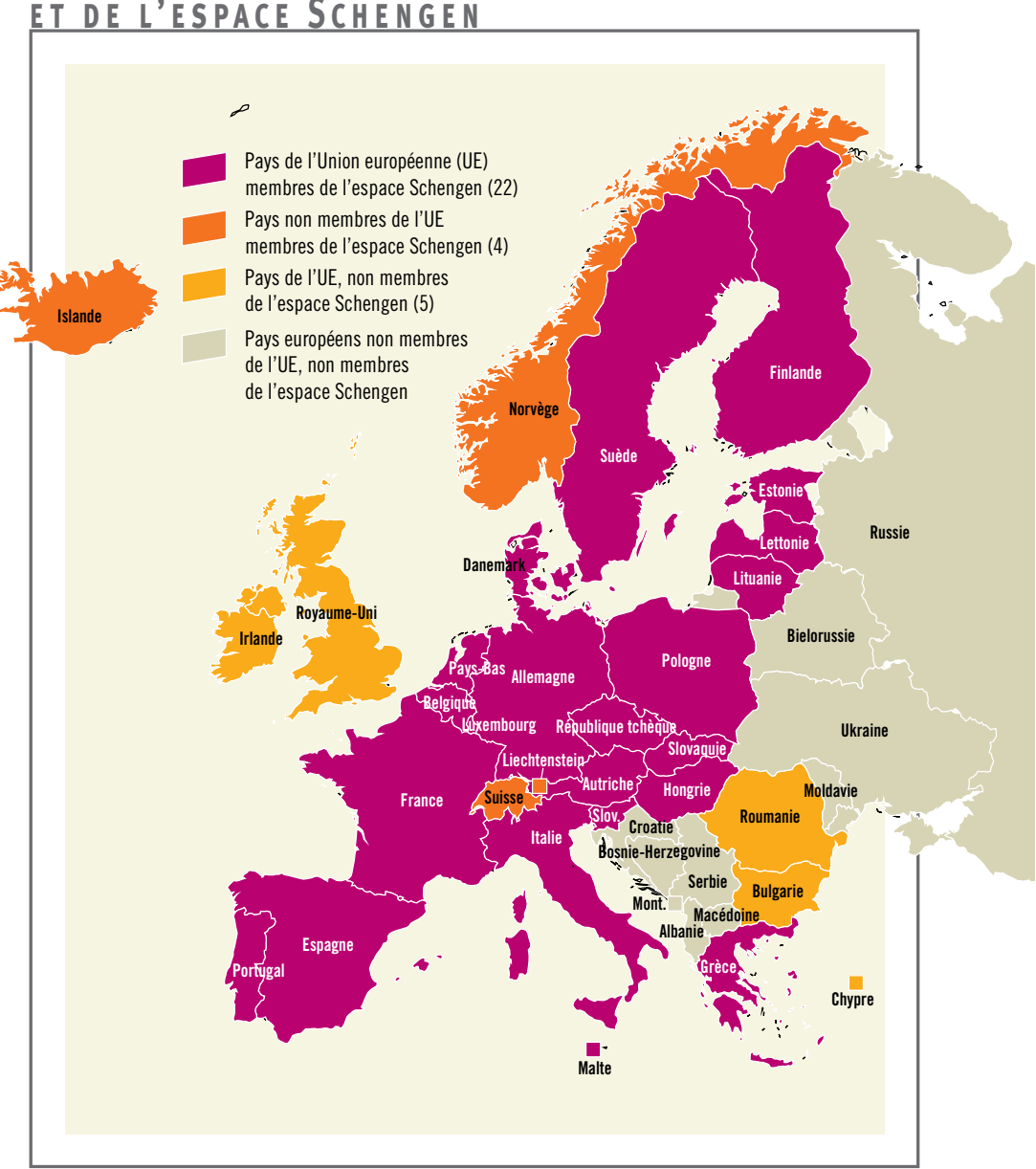

s'accompagne de quatre libertés, de libre circulation des marchandises, des services, des capitaux et des hommes, et du droit de vote aux élections municipales dans le pays de résidence, a fait largement tomber les barrières aux frontières intérieures de l'UE. Ces dernières sont encore plus abaissées en considérant seulement les pays de l'espace Schengen ou ceux de la zone euro.

Même si, selon les règles de l'ONU, la notion de frontière de ses pays membres a le même sens en terme de protection internationale, les différentes décisions géopolitiques prises par les pays conduisent à donner à leurs frontières une intensité fort différente pouvant aller d'une fermeture totale à une ouverture quasi totale comme entre certains pays européens. Il en résulte que la notion même de migrations internationales, qui implique certes le passage d'une frontière internationale, $a$ un sens fort différent selon le type de frontière concerné. Entre des pays européens à la fois membres de l'UE, de l'espace Schengen et de la zone euro, parler de "migrations internationales» peut être considéré comme excessif, tant les conditions de la migration s'apparentent davantage à une migration interne qu'à une migration internationale.

Le surgissement au sein de l'Europe de nouveaux types de frontières nés de choix politiques conduit donc à repenser l'étude des migrations internationales.
9. Imitée dans de nombreuses autres régions du monde, que l'on songe à l'Asean (Association des nations de l'Asie du Sud-Est créée en 1967 à Bangkok, allant de la Birmanie aux Philippines) ou au Consel Philippines) ou au Conseil arabes du Golfe.

10. Ainsi que l'Irlande, Chypre, la Roumanie et la Bulgarie. En outre, les territoires suivants ne font perritoires suivants ne font Schengen : les départements et territoires français d'outre-mer, les îles néerlandaises des Caraïbes, les villes de les îles Féroé et le Groënland (Danemark) et les îles Svalbard (Norvège) En rard (Norvège). En revanch I archipel des Açores l'espace Schengen. Marin et la Vatican sont de facto intégrés à l'espace Schengen. pas partie de l'espace Ceuta et Melila (Espagne), (Portugal) fait partie Enfin, Monaco, Saint- 